\title{
On some applications of one wave equation with variable coefficients
}

\author{
Senitskiy A.Yu., Evdokimova N.N. \\ Samara State Transport University
}

\begin{abstract}
In the present study we consider the cases of integrability of hyperbolic equation with variable coefficients. For this purpose, a Fourier transform is used in combination with a special representation of the transform in image space.

Various versions of closed solutions are formulated with the help of introduced arbitrary functions. The solutions obtained in the work are absent in the known reference manuals on differential equations, and the results obtained for continuously-heterogeneous anisotropic media with cylindrical or spherical symmetry at certain ratios of elastic constants of the material complement the well-known studies of wave processes in similar media [4,5] .
\end{abstract}

Keywords: hyperbolic equations with variable coefficients, Fourier transform, continuously inhomogeneous anisotropic medium, wave processes.

Citation: Senitskiy A.Yu., Evdokimova N.N. On some applications of one wave equation with variable coefficients. Proceedings of Information Technology and Nanotechnology (ITNT-2015), CEUR Workshop Proceedings, 2015; 1490: 227-233. DOI: 10.18287/1613-0073-2015-1490-227-233

In this work the procedure of building the general solution of hyperbolic equations of general form is presented, which is very effective, along with the methods of transformation of equations which use the group analysis [1,2] and methods of factorization [3].

\section{Construction of closed solutions}

Let us consider the following differential equation in the area $\Omega:\{t>0,1<r<a\}$ :

$\frac{\partial^{2} U(r, t)}{\partial r^{2}}-\frac{\partial^{2} U(r, t)}{\partial t^{2}}+A(r) \frac{\partial U(r, t)}{\partial r}+B(r) U(r, t)=0$,

where $A(r), B(r) \in C_{[1, a]}$.

We assume that function $U(r, t)$ satisfies Dirichlet conditions, therefore it can be represented by a Fourier integral, recorded as formulas: 


$$
\begin{aligned}
& \tilde{U}(r, p)=\frac{1}{\sqrt{2 \pi}} \int_{0}^{+\infty} U(r, t) e^{-i p t} d t ; \\
& U(r, p)=\frac{1}{\sqrt{2 \pi}} \int_{0}^{+\infty} \tilde{U}(r, t) e^{i p t} d p .
\end{aligned}
$$

We apply transformation (2) to (1), assuming that $U(r, 0)=\frac{\partial U(r, 0)}{\partial t}=0$. Then in the space of images we obtain the following equation:

$$
\frac{\partial^{2} \tilde{U}(r, p)}{\partial r^{2}}+A(r) \frac{\partial \tilde{U}(r, p)}{\partial r}+\left[B(r)+p^{2}\right] \tilde{U}(r, p)=0 .
$$

The solution of the equation is represented in the form:

$$
U(r, p)=\varphi(r) \cdot G(s), s=p \psi(r),
$$

where $\varphi(r), \psi(r) u G(s)$ are twice continuously-differentiable functions of their arguments. As a result of setting (5) in (4), we obtain the differential relation

$$
\begin{aligned}
& s^{2}\left[\frac{d^{2} G}{d s^{2}}+\frac{1}{\left(\psi^{\prime}\right)^{2}} G\right]+s\left[\frac{\psi^{\prime \prime} \psi}{\left(\psi^{\prime}\right)^{2}}+\frac{2 \varphi^{\prime} \psi}{\varphi \psi^{\prime}}+\frac{A \psi}{\psi^{\prime}}\right] \frac{d G}{d s}+ \\
& +\frac{\psi^{2}}{\varphi\left(\psi^{\prime}\right)^{2}}\left[\varphi^{\prime \prime}+A \varphi^{\prime}+B \varphi\right] G=0,
\end{aligned}
$$

which can be satisfied in various ways. In works $[6,7]$ we obtained closed solutions of equation (1), containing the wave functions. Let us consider an alternative option of building the general solution of equation (1) that does not contain wave functions, assuming that $\psi^{\prime}(r)=1$, i.e.

$$
\psi(r)=r \text {. }
$$

Case1.

Suppose we have the following equations:

$$
\begin{aligned}
& \frac{\psi^{\prime \prime}(r) \cdot \varphi(r)}{\left[\psi^{\prime}(r)\right]^{2}}+2 \frac{\psi(r) \cdot \varphi^{\prime}(r)}{\varphi(r) \psi^{\prime}(r)}+\frac{A(r) \cdot \psi(r)}{\psi^{\prime}(r)}=0 ; \\
& \frac{\psi^{2}(r)}{\varphi(r)\left[\psi^{\prime}(r)\right]^{2}}\left[\varphi^{\prime \prime}(r)+A(r) \varphi^{\prime}(r)+B(r) \varphi(r)\right]=-6 ;
\end{aligned}
$$

In this case, equation (6) is converted to the following form $s^{2} \frac{d^{2} G(s)}{d s^{2}}+\left(s^{2}-6\right) G(s)=0$.

The general solution of the latter equation can be represented as follows [8]:

$$
G(s)=C_{1}(p)\left\{\frac{3}{s} \cos \left[s+C_{2}(p)\right]+\left(1-\frac{3}{s^{2}}\right) \sin \left[s+C_{2}(p)\right\} .\right.
$$


Performing convertion equations (5), (10), we find

$$
\begin{aligned}
& U(r, t)= \\
& =\frac{\varphi(r)}{\sqrt{2 \pi}} \int_{0}^{+\infty} C_{1}(p)\left\{\frac{3}{s} \cos \left[s+C_{2}(p)\right]+\left(1-\frac{3}{s^{2}}\right) \sin \left[s+C_{2}(p)\right\} e^{i p t} d p .\right.
\end{aligned}
$$

From (8) correlation we find

$$
\varphi(r)=\exp \left[-\frac{1}{2} \int A(r) d r\right] \text {. }
$$

Finally, from equation (9) we obtain

$$
B(r)=\frac{1}{4}\left[2 A^{\prime}(r)+A^{2}(r)-\frac{24}{r^{2}}\right] \text {. }
$$

Theorem I.

If the coefficients of equation (1) satisfy correlation (13), then expressions (11) and (12) are its general solution.

\section{Case 2.}

Suppose that condition (7) is met and following correlations are valid:

$$
\frac{\psi^{\prime \prime}(r) \cdot \psi(r)}{\left[\psi^{\prime}(r)\right]^{2}}+\frac{A(r) \cdot \psi(r)}{\psi^{\prime}(r)}+2 \frac{\psi(r) \cdot \varphi^{\prime}(r)}{\varphi(r) \psi^{\prime}(r)}=1 ;
$$

$\frac{\psi^{2}(r)}{\varphi(r)\left[\psi^{\prime}(r)\right]^{2}}\left[\varphi^{\prime \prime}(r)+A(r) \varphi^{\prime}(r)+B(r) \varphi(r)\right]=-v^{2}$;

where $v \in R$.

Then (6) is transformed into Bessel equation, i.e.

$$
s^{2} \frac{d^{2} G(s)}{d s^{2}}+s \frac{d G(s)}{d s}+\left(s^{2}-v^{2}\right) G(s)=0 .
$$

Its solution is written as

$$
G(s)=C_{1}(p) I_{v}(s)+C_{2}(p) Y_{v}(s) .
$$

Here $I_{v}(s), Y_{v}(s)$ - Bessel function of $" v "$ order of I and II kind.

Performing conversion of expression (5), taking into account (16), we determine $U(r, t)$.

We have

$$
U(r, t)=\frac{\varphi(r)}{\sqrt{2 \pi}} \int_{0}^{+\infty}\left[C_{1}(p) I_{v}(s)+C_{2}(p) Y_{v}(s)\right] e^{i p t} d p .
$$

Taking into consideration (7), from (14) we find function $\varphi(r)=r^{1 / 2} \exp \left[-\frac{1}{2} \int A(r) d r\right]$. 
Further, from (15) it follows that

$$
B(r)=\frac{1}{4}\left[2 A^{\prime}(r)+A^{2}(r)+\frac{1-4 v^{2}}{r^{2}}\right] \text {. }
$$

Theorem II.

When condition (19) is met, expression (17) is a closed solution of equation (1).

\section{Problem statement}

The differential equation of motion of continuously-heterogeneous anisotropic elastic medium in the case of its axis-symmetric deformation, as well as the equations of state, connecting components of the stress tensor and the displacement vector, are recorded as follows [9].

$$
\begin{aligned}
& \frac{\partial \sigma_{r r}^{*}}{\partial r^{*}}+\frac{n\left(\sigma_{r r}^{*}-\sigma_{\theta \theta}^{*}\right)}{r^{*}}-\rho_{*} \frac{\partial^{2} U^{*}}{\partial t_{*}^{2}}=0, \\
& \sigma_{r r}^{*}=c_{11}^{*} \frac{\partial U^{*}}{\partial r^{*}}+n c_{12}^{*} \frac{U^{*}}{r^{*}}, \\
& \sigma_{\theta \theta}^{*}=\sigma_{y y}^{*}=c_{12}^{*} \frac{\partial U^{*}}{\partial r^{*}}+\left[c_{22}^{*}+(n-1) c_{23}^{*}\right] \frac{U^{*}}{r^{*}} .
\end{aligned}
$$

Here $\sigma_{r r}^{*}\left(r^{*}, t^{*}\right), \sigma_{y y}^{*}\left(r^{*}, t^{*}\right), \sigma_{\theta \theta}^{*}\left(r^{*}, t^{*}\right)$ are the relevant components of the normal stresses;

$U^{*}\left(r^{*}, t^{*}\right)$ are the radial component of the displacement vector;

$c_{i k}^{*}\left(r^{*}\right), \rho_{*}\left(r^{*}\right)$ are respectively elastic characteristics and density of heterogeneous anisotropic medium;

$r^{*}, t^{*}$ are radial coordinate and time; $n=1,2$ is value, corresponding to the cylindrical and spherical cavities.

After substitution of correlations (21) into (20) and introducing the dimensionless quantities by formulas

$$
\begin{aligned}
& c_{i k}=\frac{c_{i k}^{*}}{a_{33}^{*}}, r=\frac{r^{*}}{a}, U=\frac{U^{*}}{a}, \rho=\frac{\rho^{*}}{\rho_{0}}, t=\frac{1}{a} \sqrt{\frac{a_{33}^{*}}{\rho_{0}}} t^{*}, \\
& \sigma_{r r}=\frac{\sigma_{r r}^{*}}{a_{33}^{*}}, \sigma_{\theta \theta}=\frac{\sigma_{\theta \theta}^{*}}{a_{33}^{*}}, \sigma_{y y}=\frac{\sigma_{y y}^{*}}{a_{33}^{*}} .
\end{aligned}
$$

Here $a$ is the cavity radius; $a_{33}^{*}, \rho^{*}$ are corresponding stiffness coefficients of density of homogeneous anisotropic medium. Equation (20) and correlation (21) in area $\Omega:\{t>0,1<r<a\}$ are defined by the system of equations 


$$
\begin{aligned}
& \frac{\partial^{2} U(r, t)}{\partial r^{2}}+A(r) \frac{\partial U(r, t)}{\partial r}+ \\
& +B(r) U(r, t)-C(r) \frac{\partial^{2} U(r, t)}{\partial t^{2}}=0, \\
& \sigma_{r r}(r, t)=c_{11}(r) \frac{\partial U(r, t)}{\partial r}+n c_{12}(r) \frac{U(r, t)}{r}=0, \\
& \sigma_{y y}(r, t)=\sigma_{\theta \theta}(r, t)=c_{12}(r) \frac{\partial U(r, t)}{\partial r}+\left[c_{22}(r)+(n-1) c_{23}(r)\right] \frac{U(r, t)}{r},
\end{aligned}
$$

where

$$
\begin{aligned}
& A(r)=\frac{1}{c_{11}}\left[\frac{d c_{11}(r)}{d r}+\frac{c_{11}(r)}{r} n\right]=\frac{d}{d r}\left[\ln \left(c_{11} r^{n}\right)\right] \\
& B(r)=\frac{n}{c_{11}(r) r^{2}}\left[(n-1)\left(c_{12}(r)-c_{23}(r)\right)-c_{22}(r)+r \frac{d c_{12}(r)}{d r}\right], \\
& C(r)=\frac{\rho(r)}{c_{11}(r)} .
\end{aligned}
$$

If at $\mathrm{t}=0$, the elastic medium is at standstill, then

$U(r, 0)=\frac{\partial U(r, 0)}{\partial t}=0,1 \leq r<\infty$.

Note. Suppose $C(r)=\frac{\rho(r)}{c_{11}(r)}=1$. This corresponds to a constant speed of propagation of elastic waves in anisotropic heterogeneous medium. Consequently, the differential equation (22) of hyperbolic type models wave processes propagating at finite speed.

Case $1^{*}$.

From comparing the respective equations (24) and (13), it follows: Theorem III.

Expression (11) defines dynamic displacements, and by formulas (23) and heterogeneous anisotropic medium stress, if its elastic characteristics $c_{i k}(r)$ satisfy the functional equation

$$
\begin{aligned}
& 2 \frac{d^{2}}{d r^{2}}\left[\ln \left(c_{11} r^{n}\right)\right]+\left\{\frac{d}{d r}\left[\ln \left(c_{11} r^{n}\right)\right]\right\}^{2}-\frac{24}{r^{2}}= \\
& =\frac{4 n}{c_{11} r^{2}}\left[(n-1)\left(c_{12}-c_{23}\right)-c_{22}+r \frac{d c_{12}}{d r}\right] .
\end{aligned}
$$

Let us consider as an example a heterogeneous anisotropic medium, the elastic characteristics of which are periodic functions of the radial coordinate, i.e., 
$c_{11}(r)=a_{11} \sin m r, c_{12}(r)=a_{12} \sin m r$,

$c_{23}(r)=a_{23} \sin m r, c_{22}(r)=a_{22} r^{2}\left(\sin m r+\frac{1}{\sin m r}\right)$.

After substitution of equations (27) in the criterion formula (26) and simple algebraic transformations, we obtain relations connecting the elastic constants of the material

$a_{12}=\frac{a_{11}}{2}, a_{22}=\frac{m^{2}}{4 n} a_{11}, a_{23}=\frac{n^{2}+24}{4 n(n-1)} a_{11}$.

Thus, in the case of a sinusoidal law of heterogeneity of elastic and inertial characteristics of the anisotropic material (27), solution (11) is valid only for a spherical cavity $(\mathrm{n}=2)$. At that the elastic constant is chosen arbitrarily.

Case $2^{*}$.

Comparing the respective equations (24) and (19), it follows: Theorem IV.

In order for expression (17) to represent a closed solution of the considered dynamic problem for an anisotropic heterogeneous medium, it is enough to meet the functional relation, connecting characteristics $c_{i k}(r)$

$$
\begin{aligned}
& 2 \frac{d^{2}}{d r^{2}}\left[\ln \left(c_{11} r^{n}\right)\right]+\left\{\frac{d}{d r}\left[\ln \left(c_{11} r^{n}\right)\right]\right\}^{2}+\frac{1-4 v^{2}}{r^{2}}= \\
& =\frac{4 n}{c_{11} r^{2}}\left[(n-1)\left(c_{12}-c_{23}\right)-c_{22}+r \frac{d c_{12}}{d r}\right] .
\end{aligned}
$$

Suppose that characteristics of material $c_{i k}(r)$ are changed by the power law

$$
c_{11}(r)=a_{11} r^{m}, c_{12}(r)=a_{12} r^{m}, c_{23}(r)=a_{23} r^{m}, c_{22}(r)=a_{22} r^{m} \text {. }
$$

Here $\mathrm{m}$ is an index of anisotropic medium heterogeneity;

$a_{i j} ; i, j=1,2,3$ are dimensionless constants of anisotropic material.

Then relation (29) takes the form

$$
v^{2} a_{11}=n\left[(n-1) a_{23}+a_{22}\right]-[n(n-1)+m] a_{12} \text {. }
$$

From (31) it follows that three of the four parameters $a_{i k}$ can be selected arbitrarily. If the elastic characteristics of the material change by the cosine law,

$$
\begin{aligned}
& c_{11}(r)=a_{11} \cos m r, c_{12}(r)=a_{12} \cos m r, \\
& c_{23}(r)=a_{23} \cos m r, c_{22}(r)=a_{22} r^{2}\left(\cos m r+\frac{1}{\cos m r}\right),
\end{aligned}
$$

the elastic constants $a_{i k}$ of the material satisfy relations

$$
a_{12}=\frac{a_{11}}{2}, a_{22}=\frac{m^{2}}{4 n} a_{11}, a_{23}=\frac{1}{4}\left[1-\frac{1-4 v^{2}}{n(n-1)}\right] a_{11} \text {. }
$$


Thus, in this case, only one elastic constant of the anisotropic material is arbitrary.

\section{References}

1. Zaytsev VF, Polyanin AD. Handbook on nonlinear differential equations. Moscow: Nauka, 1993; 462 p. [in Russian]

2. Zaytsev VF. Discrete-group analysis of ordinary differential equations. Differential equations, 1989; 25(3): 379-387. [in Russian]

3. Berkovich LM. Factorization and transformations of ordinary differential equations. Edited by N.K. Rozova. Saratov: Publishing House of Saratov University, 1989; 192 p. [in Russian]

4. Petrashen GI. Wave propagation in anisotropic elastic media. Leningrad: Nauka, 1980; 280 p. [in Russian]

5. Green W. Wave propagation in strongly anisotropic elastic materials. Arch.Mech.Stosow, 1978; 30(3): 297-307.

6. Senitskiy AY. On the integrability of the wave equation with variable coefficients. Proceedings of the higher educational institutions. Mathematics, 1998; 7: 39-46.

7. Evdokimova NN, Senitskiy AY, Kharkovskiy SI. The study of wave propagation in infinite anisotropic media. Int. scientific conference "Modern problems of mathematics, mechanics, computer science." Proceedings of the conference. Tula: TSU, 2007; 145-147.

8. Kamke E. Handbook on ordinary differential equations. Moscow: Nauka, 1965; 703 p. [in Russian]

9. Lyav A. Mathematical theory of elasticity. Moscow: ONTY, 1935; 674 p. [in Russian] 